\title{
THE EFFECTS OF PERCEPTIONS OF HAZARDOUS WASTE ON MIGRATION: A LABORATORY EXPERIMENTAL APPROACH
}

\author{
Michael J. Greenwood, Gary H. McClelland, and William D. Schulze*
}

\begin{abstract}
This study develops a methodology that allows migration decision-making to be studied in a laboratory experimental setting. Moreover, this methodology permits an examination of the importance of natural and man-made hazards in migration decisions-factors that have not been extensively studied as determinants of migration. The specific application is to the location of the U.S. nuclear waste repository at Yucca Mountain, Nevada. Empirical results suggest that the repository may influence employment-related migration, but probably not retirement migration.
\end{abstract}

\section{INTRODUCTION}

Although many migration determinants have been studied in some detail, the importance of potential natural hazards like earthquakes and the influence of manmade hazards like the presence of a nuclear waste facility have not been addressed in a carefully designed, scientific study. ${ }^{1}$ Moreover, subjective beliefs about risk may exert an important influence on migration decisions, but to our knowledge these types of migration determinants have not been studied. The objective of this paper is to partially rectify this gap in knowledge. Using laboratory experimental techniques, we study the influence of risks associated with the transportation and storage of nuclear waste materials on potential migration. Our specific application is the proposed national high-level nuclear waste site at Yucca Mountain, Nevada, but the methodology we develop and apply in this case study also is applicable to other situations regarding natural and man-made hazards.

The siting of a facility such as the proposed nuclear waste repository at Yucca Mountain may influence the decisions of people to migrate to the geographic region in which the facility is located. It is of course impossible to assess the actual behavioral effect of a facility on migration decisions prior to the

\footnotetext{
* Department of Economics and Center for Economic Analysis, University of Colorado at Boulder; Department of Psychology and Center for Research on Judgment and Policy, University of Colorado at Boulder; and Kenneth L. Robinson Professor of Agricultural Economics and Public Policy, Department of Agricultural, Resource, and Managerial Economics, Comell University, respectively. This research was supported by the Yucca Mountain Socioeconomic Project, State of Nevada. The authors are grateful to David J. Schenk, Steven R. Elliot, and David R. McCormick for assistance. Paul Slovic and Howard Kunreuther made a number of helpful suggestions during the course of the study, for which the authors are also grateful.
} 
siting of the facility. The next best alternative is to study the psychological decision process used to make migration decisions and to determine experimentally how the existence of a particular facility would impact those decisions. Once the impact on the decision process is determined, it is possible to predict how the current migration flow to the geographic region would be influenced by the siting of the facility.

In this study, such an assessment is made in the context of a psychological decision process that is traced through the use of a computer program that allows individual subjects to sift through a large quantity of information about alternative areas to which they might migrate. Subjects are free to examine some or all available information, but are not "forced" to consider any given item. After considering the information they wish to view, the subjects choose a location where they wish to migrate. Then their choices are analyzed.

In the following section we describe how the impact of a facility siting on migration decisions can be measured. The particular focus in the discussion is on the migration decisions of people who have retired or who are about to retire. However, the same basic assessment strategy also is applied to the migration decision process of those still in the labor force. Indeed, much of the work described in later sections refers to migration decisions of persons of labor-force age who have relatively high propensities to make interstate moves. We provide a fairly detailed discussion of the precise methodology developed for this study, including a description of the computer protocol, recruitment of subjects, and the actual implementation of the choice experiment. Then we present the empirical findings, as well as discuss possible extensions of the work.

\section{BACKGROUND}

\section{Identification of Decision Components}

The first step is the identification of the important components or factors that people consider when deciding if to migrate, and if so, where to migrate. Existing demographic and economic studies of migration flows can be used to suggest components for the list. Obvious components for retirement migration decisions are living costs, climate, whether family and friends live in the area, opportunities for part-time employment, geographic terrain, and cultural resources. ${ }^{2}$ Of course, one component that would be included automatically in this study would be the presence of disamenities such as a nuclear waste repository. The regular appearance of trucks carrying nuclear waste also could be included on this list. Furthermore, some attention could be given to a worst-case accident involving either 
the transportation or the storage of waste materials, but we did not conduct any such experiment.

\section{Identification of the Alternative Set}

An axiom of choice theory is that it is not the absolute evaluation of an alternative but rather the relative comparison of an alternative against its competitors that determines choice. That is, one option for a retirement location might receive very high absolute evaluations but still be seldom chosen because another slightly better retirement location exists. This issue may be particularly important regarding the impact of facility citing because an undesirable facility may not reduce the absolute evaluation of a particular retirement location very much, but just enough to tip the balance in favor of alternative retirement locations. ${ }^{3}$ In the methodology described below, several western and southwestern metropolitan areas constitute the alternative set.

\section{Assessment of Beliefs about Retirement Locations}

Each prospective retirement location may be viewed as a bundle of components or attributes. People's beliefs about these components may be inaccurate. For example, a retired couple in New Jersey considering a move to Nevada or some other state may have a distorted image of the climate and geography of particular retirement locations. Thus, beliefs about the components, and not the actual components, will have a direct impact on decision processes. In particular, people's beliefs about the location and effects of a nuclear waste repository, and not the actual location and effects, will influence decisions to move to a region containing the repository. 4

\section{Assessment of the Decision Process}

The literature on judgment and decision-making suggests two broad classes of psychological decision models: compensatory and noncompensatory or hierarchical (Abelson and Levi 1985; Einhorn 1970; Einhorn, Kleinmuntz, and Kleinmuntz 1979; Montgomery and Svenson 1976; Tversky 1972; Tversky and Sattath 1979). In compensatory models, which are consistent with utility theory, benefits are traded off psychologically against costs. For example, a retiree may decide to accept certain disamenities such as climate or the presence of an undesirable facility in order to get the benefits of lower living costs and lower taxes. In compensatory models, no matter how great the disamenities of a retirement location, the location still may be chosen because it has other desirable attributes 
that outweigh the disamenities. In noncompensatory models, components or attributes are considered serially or hierarchically so that the existence of a serious undesirable attribute is sufficient to eliminate a prospective retirement location from consideration without an individual ever considering the positive aspects of that location. If the presence of a nuclear waste repository were sufficient to reject a retirement location from further consideration, the consequences on decisions to migrate to a region in which such a facility were located would be dramatic. In the work described below, these two broad classes of decision models are studied in the context of migration decision making. The strategies for each class of decision model are described separately.

Compensatory Models. The issue is how much of an undesirable attribute an individual is willing to accept in order to get a given desirable attribute. Techniques for measuring such tradeoffs in compensatory models are well known (Anderson 1982; Hammond et al. 1975; Nickerson and McClelland 1984; Slovic and Lichtenstein 1971). The basic strategy is to have individuals evaluate (perhaps on rating scales) a number of alternative retirement locations, each described in terms of the components or attributes identified above. Statistical procedures such as regression, analysis-of-variance, or conjoint measurement can then be used to measure the relative importance of each attribute in the overall evaluation of retirement locations. This strategy already has been applied to the study of retirement decisions by Pampel, Levin, Louviere, Meyer, and Rushton (1984). They of course do not include an attribute for the presence of a nuclear waste repository or similar facility. Moreover, their methodology differs from ours in several other respects.

Noncompensatory Models. The issue is whether people eliminate prospective retirement locations on the basis of a very limited set of attributes and, if so, whether the presence of a nuclear waste repository would be one of those attributes. Verbal protocol or "think aloud" procedures (Ericsson and Simon, 1984) have been successful in studying noncompensatory decision processes (Bettman and Park 1980; Einhorn, et al. 1979). A small sample of retirees, tested individually in the laboratory, could be presented with a set of real retirement locations described only by their names and asked to talk aloud as they think about which of these locations they would choose. The effect of hazardous facilities in a region could be assessed by including locations that did or did not have such facilities. Another small sample of retirees could be presented with a set of hypothetical retirement locations described in terms of a bundle of attributes and asked to talk aloud as they choose among the locations. For some of the hypothetical locations, one of the attributes could be a hazardous waste facility. From the transcriptions of the recorded protocols, those attributes used to dismiss alternative locations early in the decision process may be determined. Such 
protocols usually contain statements such as "I won't consider any alternatives that have. . ." or "I will only consider alternatives that have. .." While such a study is possible, we have not undertaken it in our research.

In place of such a "case study" strategy in which each participant is treated as a separate case, we have adopted an alternative that is unique for this type of study. We have drawn a sufficiently large sample of subjects that statistical confidence can be placed in the findings.

\section{COMPUTER PROTOCOL AND PARTICIPANT SELECTION}

Our method falls within the class of "process-tracing" techniques frequently used in psychology and marketing to study decision processes (Greenwood, Mueser, Plane, and Schlottmann 1991). It is important to realize that the purpose of these techniques is to understand the decision process rather than to predict decision outcomes for a large sample of people. As a consequence, our methods are much more intensive than extensive. That is, we collect much detailed process information from a relatively small sample of people. However, as pointed out above, we wish to go somewhat beyond current procedures in this area of research by increasing sample size sufficiently that statistical confidence can be placed in the results. The importance of this phase of our research effort lies in the development of a methodology and its application to migration decision-making.

Intensive details of interest are the order in which people consider relevant information when making a decision and the amount of time they spend with each piece of information. ${ }^{5}$ Keeping track of such details requires that data collection be computer-controlled. In the laboratory studies the computer displays all stimulus information and relevant questions and records-not only the participant's responses, but also all the detailed process information. Below we describe the basic data collection procedure. Several variations of this basic procedure are described later.

\section{The Decision Components}

As discussed previously, several decision components had to be operationalized for each metropolitan area in the alternative set. These components are essentially characteristics of the areas that are expected to enter importantly into the migration decision process. Based upon an extensive review of the migration literature, we settled on five "menus": 1) economic conditions, 2) climate and terrain, 3) recreation, culture, and education, 4) hazards, environment, and safety, and 5) people and friends. 
With two exceptions, each menu contains factual information about a specific metropolitan region. Since we have no convenient way of knowing beforehand whether subjects have family and/or friends living in any specific metropolitan area, the people and friends menu contains suppositions, such as "you have no close relatives or friends living in this area." However, this menu contains factual information pertaining to racial/ethnic composition of each area's population. The second exception relates to the nuclear waste repository and is discussed below.

Because an enormous quantity of information is available for review by each subject, a "sub-menu" design was used in the computer protocol. This design breaks each menu into categories (that might be called "cards" because the computer protocol mimics flash cards). The submenus are 1) economic conditions: a. employment; b. cost of living; 2) climate and terrain: a. climate; b. terrain, 3) recreation, culture, and education: a. recreation; b. culture; c. education; 4) hazards, environment, and safety: a. natural hazards; b. technological hazards; c. pollution; d. crime; and 5) people and friends: a. people; b. friends. Thus, for each metropolitan area 13 "cards" are available for review by participants in the experiment.

A detailed list of information for each metropolitan area included in the study is available from the authors on request. However, since the focus of the research is on the effects of a nuclear waste repository, we briefly consider the "technological hazards" submenu here. Except for the nuclear waste repository scenario, this submenu also contains factual information. The nuclear waste repository scenario with which we worked reads as follows:

A high-level nuclear waste repository is located 100 miles west of City X. Waste materials will be regularly shipped through City $\mathbf{X}$ to the repository. Several small accidents could occur each year and could involve worker exposure to radiation.

A serious accident involving a fire is unlikely, but could occur and cause contamination of repository buildings, radiation contamination of the air, and hospitalization of some workers due to radiation exposure.

The media may raise questions about management and safety at the repository, and health concerns may be raised for people living near the site.

As discussed below, this scenario was placed in different metropolitan regions, and not just Las Vegas, which is the closest metropolitan area to Yucca Mountain. 


\section{The Set of Alternatives}

Previously we pointed out the importance of specifying an alternative set of destination choices that might be selected by decision-makers. In the absence of a survey to determine what areas constitute viable alternatives for potential migrants to Las Vegas, we decided to use several roughly comparable western and southwestern metropolitan areas. In the actual computer protocol, these areas are never specifically identified. Rather, they are referred to by letters as follows: City A: Albuquerque; City B: Denver; City C: El Paso; City D: Las Vegas; City E: Los Angeles; City F: Phoenix; City G: Salt Lake City; City H: San Diego; City I: Tucson. The computer protocol therefore contained 117 "cards" ( 9 areas $\mathrm{x}$ 13 submenus). ${ }^{6}$

\section{Participants}

Subjects who were to work through the computer protocol were recruited through a two-step procedure. The first step involved an examination of published migration data relating to both the State of Nevada and Clark County, Nevada, and, in light of these data, the second step involved the actual recruitment of subjects. The migration data, which were primarily drawn from 1980 U.S. Census information, were important in allowing the study team to learn whether any specific characteristics of migrants to Nevada warranted attention.

Just over 40 percent of Nevada's 1975-80 in-migrants were between 20 and 30 years of age, which suggests a strong orientation toward individuals with the highest propensities to migrate nationwide. Numerous studies suggest that a primary determinant of the migration of persons in this general age class is job opportunities. Thus, one broad group of participants was oriented toward the age class roughly between 25 and 35 .

A second broad group on which attention focused was individuals in their pre-retirement years. While only about 6 percent of Nevada's 1975-80 inmigrants were 65 years old and over, some potential for substantial retirement migration to Las Vegas may exist because of the strong orientation toward recreational opportunities there. Because the act of having made a retirement move could affect an individual's decision process, we settled on a group of individuals roughly in pre-retirement years, or about 55 to 65 years old.

Subjects to participate in the experiment were recruited from three sources, namely, from among University of Colorado staff members, from off-campus, and from University of Colorado graduate students. Most subjects were contacted by telephone, but some were contacted in person. A total of six different specific groups participated in the experiment, which was conducted in three waves. The 
first wave of the experiment was conducted during April and May, 1988. The second wave was conducted during August, 1989, and the third wave during November, 1989. The first wave of subjects consisted of four groups. Group 5 was the entire second wave, and group 6 was the entire third wave.

Group 1 was a control group made up of 29 persons drawn from the University of Colorado staff and from off-campus through personal contacts. The choice problem given to the control group contained no nuclear repository in any metropolitan area. The idea was to use the control group to identify the most popular city or cities. Then for subsequent groups, a repository would be placed in one or more popular cities. If the location of the repository in such cities affected migration choices, this outcome ought to have been evident for subsequent groups in the sense that their location choices would differ in a systematic way from those of the control group. For several subjects, the repository was also left in Las Vegas.

Group 2 was comprised of 16 University of Colorado staff members and other individuals. Group 3 was made up of 16 University of Colorado staff members who were nearing the age of retirement (i.e., aged approximately 55 to 65). Group 4 consisted of 32 persons drawn from University of Colorado staff and graduate students, as well as other individuals. Each group was administered the protocol on a different evening. Groups 2,3 , and 4 considered choices that included a nuclear waste repository. Groups 2 and 3 dealt with choices that had a repository near City D (Las Vegas) or City H (San Diego), but not both D and H. As subjects from Groups 2 and 3 arrived for the experiments, they were randomly assigned to a protocol that had a repository in one or the other of these cities. Group 4 was faced with a repository in City $\mathbf{H}$. A total of 16 subjects had a repository near City D ( 9 from the retirement group and 7 from among those making job choices) and 48 had a repository near City $\mathrm{H}$ (8 from the retirement group and 40 from among those making job choices). One individual from Group 4 was assigned to the retirement group because of age, bringing the total number of subjects making a retirement decision to 17. A grand total of 93 subjects participated in the initial phase of the experiment.

Group 5 consisted of 82 subjects, all of whom were confronted with a repository in City H. These subjects were tested in August, 1989, approximately one year after the initial 93 subjects were processed. The reason for using the 82 additional subjects was to increase sample size in an effort to get more people in the subject pool who actually looked at the repository card. Moreover, additional funding was required before this and the subsequent group were processed.

Group 6, which was processed in November, 1989, consisted of 58 subjects. This group also was presented with a repository in City $\mathrm{H}$, but the instructions provided at the beginning of the computer protocol were different. Groups 1 
through 5 were not given contextual information concerning the experiments, whereas Group 6 was told that the study was about the effect of locating a highlevel nuclear waste repository in a western state. The idea was to see if this group was more likely to examine the repository card than those that were not informed about the goals of the study. Unlike groups 1-4, no special effort was made to restrict groups 5 and 6 to the two ages classes that were the focus of attention during the first phase of the study. Table 1 summarizes the information on the subjects.

TABLE 1

Treatments Across Subject Pool

\begin{tabular}{|c|c|c|c|c|c|c|}
\hline Group & $\begin{array}{l}\text { Number } \\
\text { of } \\
\text { Subjects }\end{array}$ & $\begin{array}{c}\text { Retirement } \\
\text { Location } \\
\text { Decision } \\
\end{array}$ & $\begin{array}{c}\text { Job } \\
\text { Location } \\
\text { Decision } \\
\end{array}$ & $\begin{array}{c}\text { Repository } \\
\text { in } \\
\text { City D }\end{array}$ & $\begin{array}{c}\text { Repository } \\
\text { in } \\
\text { City } \mathrm{H}\end{array}$ & $\begin{array}{l}\text { Highlight } \\
\text { Repository in } \\
\text { Intructions }\end{array}$ \\
\hline $\begin{array}{l}1 \\
2 \\
3 \\
4 \\
5 \\
6\end{array}$ & $\begin{array}{l}29 \\
16 \\
16 \\
32 \\
82 \\
58\end{array}$ & $\begin{array}{l}\ddot{1} \\
16 \\
- \\
\ddot{=}\end{array}$ & $\begin{array}{l}29 \\
15 \\
32 \\
82 \\
58\end{array}$ & $\begin{array}{l}\overline{8} \\
8 \\
8 \\
- \\
=\end{array}$ & $\begin{array}{c}8 \\
8 \\
32 \\
82 \\
58 \\
\end{array}$ & $\begin{array}{l}\bar{E} \\
\overline{58} \\
\overline{58}\end{array}$ \\
\hline Total & 233 & 17 & 216 & 16 & 188 & 58 \\
\hline
\end{tabular}

Thus, for the first wave of experiments, two groups of subjects were studied, those considering a place to retire and those considering a place to work. As pointed out above, the retirement group was roughly aged 55 to 65 and the employment group was roughly aged 25 to 35 . We do not claim that the sample of individuals chosen for study is a representative, random sample. Clearly, it is not. Several sources of bias may enter the decision process. For example, to the extent that migration involves a process of selection, individuals who prefer Boulder, Colorado, may settle there, whereas those who prefer, say, Los Angeles, settle in L.A. Thus, individuals residing in different parts of the country may make final destination choices that differ systematically from those of our Boulder subjects. However, in this study we are interested in the methodology, and thus the process by which individuals sort through complex information is, in many respects, more important to us than their actual choices.

\section{Implementation of the Experiment}

The location choice experiment was implemented through the use of an interactive program applied and developed by the investigators. The experiment was designed in such a way that a computer illiterate person could comfortably sit down and work through the program without the added distraction of worrying about how to operate it. After the subjects were seated and had read and signed a consent form, they were directed to begin the experiment that required them to go 
through an initial set of instructions on the computer. These instructions were intended to acclimate the individual to the use of the mouse and provide a dry run for them to work through in order to become familiar with the program. To allow the individual to abstract from his/her perceptions about his/her own job prospects in each city, the subject was asked to assume that an equivalent job offer with equal pay is forthcoming in each city. The final part of the introduction is the provision of a trial choice decision in which a location choice must be made between two hypothetical cities $\mathrm{X}$ and $\mathrm{Y}$, using the same procedure that is utilized in the "real" choice problem.

After the successful review and completion of the instructions and practice problem, the subject moved on to the real choice problem. The first card lays out the basic framework in which the subject operates throughout the experiment. Moreover, the subject is always given an opportunity to indicate that he/she is ready to make a final choice. To start the process, a category and a city must first be selected. Next, a third menu is added to the screen that contains the list of items available for inspection pertaining to the category and city selected. Once the selection is made, the OK button is clicked and the card with the appropriate description shows on the computer screen for the subject's perusal.

After the subject finishes a card, the DONE button is clicked and the initial display is returned to the screen. The process continues in this manner. No time limit was placed on the viewing of any single card, and each one could have been recalled any number of times and in any order. Furthermore, no single card was required to be viewed in order to make a choice among the various cities. When the final choice was to be made, the subject was required to indicate that he or she was ready, which in turn led to the presentation of the final choice menu. The final choice could be made at this time and recorded, or the individual could have gone back into the item selection mode to reconsider or gather more information.

Each person was allowed up to one hour to work through the program and make his or her choice. Some would have taken much more than an hour, if given the chance. However, when necessary, the monitor would nudge people along by suggesting that they move on from the practice problem if they were spending too much time on it. Likewise, near the end of the hour, participants were reminded that they should have been finishing and should start considering their final choices. Yet, in most cases, subjects finished making their final choice well before the end of the hour. 


\section{EXPERIMENTAL RESULTS}

\section{Types of Cards Examined}

Recall that Groups 2 and 3 participated in the first experiments. The initial findings derived from these groups are based on a sample size of 32 subjects and guided the design of research that followed. Seventeen of the initial subjects were involved in making a retirement location choice, and 15 were required to choose a job location. Table 2 indicates how much information was considered by these groups and how frequently technological hazards were employed in the choice process. Clearly, the younger, job-choice group examined more cards, on average, than did the older, retirement group. The job-choice group examined significantly more information, about 50 percent more, than the retirement group $(t(30)=2.7$, $p=.012)$. However, the numbers in the top panel of Table 2 have not been adjusted for redundancy (repeated scanning). The types of information considered by the pre-retirement and the job-related groups also may differ. For example, pre-retirement individuals may spend less time considering the employment characteristics, but more time studying cost-of-living information and information about family and friends and about recreational features of an area.

Table 2 also provides information on the average number of inquiries made regarding the nature of a technological hazard associated with any particular city. With nine cities in the choice set, a subject could potentially examine nine technological hazard cards. Moreover, any of these nine cards could be examined multiple times. Thus, in Table 2 the entry 4.6 for the 15 job location subjects indi-

TABLE 2

Cards Examined by Groups 2 and 3

Job location

Retirement location

Total

All cards examined

\begin{tabular}{lrrr} 
No. of subjects & 15 & 17 & 32 \\
Mean no. of cards examined & 64.5 & 42.5 & 52.3 \\
Percent of total cards & 59 & 39 & 48 \\
\hline
\end{tabular}

Technolopical hazard cards examined

Mean no. of cities whose card was examined per subject

Percent of cities whose card was examined

Mean no. examined adjusted for covariates 
cates that an average of 4.6 technological hazards cards were examined by members of this group. The 51 percent is formed on a base of nine cities, but again some of the subjects may have examined one or more cities multiple times. The difference between how often the hazard is considered by the two groups is significant. Individuals making a job location choice accessed almost four times more information about hazards than those facing a retirement decision $(t(30)=3.32, p=.002)$.

Finally, Table 2 provides an analysis of covariance of the number of technological hazard cards inspected, controlling for the total number of cards examined. This analysis adjusts for the fact that, if a greater number of cards is examined, the likelihood of inspecting a technological hazard card increases. When the two groups are compared in this way, the difference is slightly smaller, but still statistically reliable $(t(29)=2.2, p=.035)$.

An interesting coincidence occurred on the day that 16 of the 17 retirement choice subjects participated in the experiment. ${ }^{7}$ (This was one day after the initial group of job location subjects participated). The local and national news media had headlined the Henderson, Nevada, rocket fuel explosion of May 4, 1988, on the morning of May 5, which was the day that the retirement experiment was run. Thus, if any group had a heightened awareness of technological hazards, it would have been the retirement group. However, in spite of the Henderson incident, the older individuals gave much smaller weight to technological hazards compared to the younger individuals. This result is consistent with a priori expectations.

Based upon these initial results, we decided to expand the sample size for the job location choice because this particular group appeared to show more concern for technological hazards. Thus, 61 new job location choice subjects were added, which produced a pool of 93 subjects for analysis for the first wave of experiments. Table 3 presents information on the mean number of cards examined, by detailed type of card, for various classes of subject. Note that this table relates to all 93 subjects and not to Groups 1 and 2 only, whose pattern of card examination was described above. The data presented in Table 3 are not corrected for the total number of cards examined by each subject.

Several interesting patterns emerge from Table 3. For example, the 76 subjects who were considered potential job-related migrants viewed an average of 5.1 employment cards, compared to an average of only 1.4 such cards for the group considered potential retirement migrants. On the other hand, the older group read an average of 4.9 "friends" cards, compared to an average of 3.4 of these cards for the younger group. The older group also paid relatively more attention to cost of living and climate, whereas the younger group devoted relatively more effort to examining terrain, recreation, education, technological hazards, and pollution. 
TABLE 3

Mean Cards Examined by Detailed Subcategory and Type of Subject

\begin{tabular}{llllllllllllll}
$\begin{array}{l}\text { Type of } \\
\text { Subject }\end{array}$ & E & CL & CM & TE & RE & CU & ED & NH & TH & PO & CR & PE & FR \\
\hline Job (76) & 5.1 & 4.4 & 5.3 & 3.5 & 3.7 & 2.6 & 2.3 & 2.2 & 2.2 & 3.3 & 2.6 & 2.8 & 3.4 \\
Ret. (17) & 1.4 & 5.5 & 6.1 & 2.1 & 3.1 & 2.5 & 1.6 & 2.2 & 1.2 & 2.1 & 2.4 & 2.4 & 4.9 \\
Control (29) & 5.6 & 4.4 & 6.0 & 2.9 & 2.9 & 2.8 & 2.3 & 2.2 & 1.7 & 3.1 & 2.7 & 2.9 & 3.8 \\
Test (64) & 3.9 & 4.7 & 5.2 & 3.4 & 3.9 & 2.5 & 2.1 & 2.2 & 2.1 & 3.1 & 2.5 & 2.6 & 3.6 \\
$\quad$ Job (47) & 4.8 & 4.4 & 4.9 & 3.9 & 4.2 & 2.5 & 2.3 & 2.1 & 2.5 & 3.5 & 2.6 & 2.7 & 3.2 \\
$\quad$ Ret. (17) & 1.4 & 5.5 & 6.1 & 2.1 & 3.1 & 2.5 & 1.6 & 2.2 & 1.2 & 2.1 & 2.4 & 2.4 & 4.9 \\
City D (64) & 3.4 & 5.7 & 6.1 & 2.8 & 3.3 & 3.3 & 2.6 & 2.4 & 1.8 & 3.1 & 3.1 & 3.1 & 5.4 \\
$\quad$ Job (7) & 6.9 & 5.0 & 5.3 & 3.6 & 4.3 & 3.4 & 3.3 & 3.1 & 4.0 & 5.0 & 3.6 & 4.1 & 6.3 \\
Ret. (9) & 0.8 & 6.2 & 6.7 & 2.1 & 2.4 & 3.1 & 2.0 & 1.8 & 0.1 & 1.7 & 2.7 & 2.2 & 4.9 \\
City H (48) & 4.0 & 4.3 & 4.9 & 3.7 & 4.1 & 2.3 & 2.0 & 2.1 & 2.3 & 3.1 & 2.3 & 2.4 & 3.0 \\
$\quad$ Job (40) & 4.4 & 4.3 & 4.8 & 4.0 & 4.2 & 2.4 & 2.2 & 2.0 & 2.2 & 3.2 & 2.4 & 2.4 & 2.6 \\
Ret. (8) & 2.0 & 4.6 & 5.5 & 2.0 & 3.9 & 1.9 & 1.1 & 2.8 & 2.5 & 2.5 & 2.0 & 2.5 & 5.1 \\
\hline
\end{tabular}

Symbols: $\mathrm{E}=$ employment $\mathrm{CL}=$ cost of living, $\mathrm{CM}=$ climate, $\mathrm{TE}=$ terrain, $\mathrm{RE}=$ recreation, $\mathrm{CU}=$ culture, $\mathrm{ED}$ $=$ education, $\mathrm{NH}=$ natural hazards, $\mathrm{TH}=$ technological hazards, $\mathrm{PO}=$ pollution, $\mathrm{CR}=$ crime, $\mathrm{PE}=$ people, and FR $=$ friends.

\section{Location Choice}

Tables 4 and 5 summarize the city choices of the various classes of subjects. For example, at least one person in the Control Group selected each city, but City H (San Diego) was clearly the most popular location, followed by City G (Salt Lake City). A total of 11 Control Group subjects (37.9 percent) selected City H, and 6 Control Group subjects (20.7 percent) chose City G. Table 4 also indicates that when the nuclear waste repository was placed in City $\mathrm{H}$, only 4 of 48 subjects (or 8.3 percent) selected City $\mathrm{H}$. These 48 subjects include 8 pre-retirement and 40 job-related potential migrants. The 40 job related subjects are the more relevant to examine because the Control Group is all job-related. Of the 40 younger, job-related subjects who confronted a repository in City $\mathrm{H}, 4$ (or 10.0 percent) actually selected $H$. Table 5 disaggregates the subjects who confronted a repository in City D and in City $\mathrm{H}$, as shown in Table 4, into those of job age and those nearing retirement.

Table 6 includes only the 69 younger individuals making a job-location decision. These subjects were presented either with no repository in any city (for the control group) or with a repository in City $\mathrm{H}$. The repository was placed in City $\mathrm{H}$ because it was the most popular city for the initial group that made a job choice. By placing the repository in the most popular city, the probability that the repository would be examined was increased. 
TABLE 4

City Choice by Class of Subject

\begin{tabular}{|c|c|c|c|c|c|c|c|c|c|c|}
\hline \multirow{2}{*}{$\begin{array}{l}\text { City } \\
\text { Choice }\end{array}$} & \multicolumn{2}{|c|}{ Control Group } & \multicolumn{2}{|c|}{$\begin{array}{l}\text { Repository in } \\
\text { City D }\end{array}$} & \multicolumn{2}{|c|}{$\begin{array}{l}\text { Repository in } \\
\text { City H }\end{array}$} & \multicolumn{2}{|c|}{$\begin{array}{l}\text { Repository in } \\
\text { Either D or H }\end{array}$} & \multicolumn{2}{|c|}{ All Subjects } \\
\hline & No. & Percent & No. & Percent & No. & Percent & No. & Percent & No. & Percent \\
\hline Albuquerque(A) & 2 & 6.9 & 1 & 6.3 & 7 & 14.6 & 8 & 12.5 & 10 & 10.8 \\
\hline Denver(B) & 1 & 3.4 & 1 & 6.3 & 12 & 25.0 & 13 & 20.3 & 14 & 15.1 \\
\hline El Paso(C) & 1 & 3.4 & 1 & 6.3 & 2 & 4.2 & 3 & 4.7 & 4 & 4.3 \\
\hline Las Vegas(D) & 2 & 6.9 & 3 & 18.8 & 6 & 12.5 & 9 & 14.1 & 11 & 11.8 \\
\hline Los Angeles(E) & 3 & 10.3 & 0 & 0.0 & 3 & 6.3 & 3 & 4.7 & 6 & 6.5 \\
\hline Phoenix(F) & 1 & 3.4 & 0 & 0.0 & 0 & 0.0 & 0 & 0.0 & 1 & 1.1 \\
\hline Salt Lake City(G) & 6 & 20.7 & 4 & 25.0 & 13 & 27.1 & 17 & 26.6 & 23 & 24.7 \\
\hline San Diego(H) & 11 & 37.9 & 5 & 31.3 & 4 & 8.3 & 9 & 14.1 & 20 & 21.5 \\
\hline Tucson(I) & 2 & 6.9 & 1 & 6.3 & 1 & 2.1 & 2 & 3.1 & 4 & 4.3 \\
\hline All Cities & 29 & 100.0 & 16 & 100.0 & 48 & 100.0 & 64 & 100.0 & 93 & 100.0 \\
\hline
\end{tabular}

TABLE 5

City Choice by Class of Subject with Age Detail

\begin{tabular}{|c|c|c|c|c|c|c|c|c|}
\hline \multirow[b]{3}{*}{ Choice } & \multicolumn{4}{|c|}{ Repository in City D } & \multicolumn{4}{|c|}{ Repository in City H } \\
\hline & \multicolumn{2}{|c|}{ Job Age } & \multicolumn{2}{|c|}{ Retirement Age } & \multicolumn{2}{|c|}{ Job Age } & \multicolumn{2}{|c|}{ Retirement Age } \\
\hline & No. & Present & No. & Present & No. & Present & No. & Present \\
\hline Albuquerque(A) & 0 & 0.0 & 1 & 11.1 & 6 & 15.0 & 1 & 12.5 \\
\hline Denver(B) & 0 & 0.0 & 1 & 11.1 & 9 & 22.5 & 3 & 37.5 \\
\hline El Paso(C) & 0 & 0.0 & 1 & 11.1 & 2 & 5.0 & $\mathbf{0}$ & 0.0 \\
\hline Las Vegas(D) & 1 & 14.3 & 2 & 22.2 & 5 & 12.5 & 1 & 12.5 \\
\hline Los Angeles(E) & 0 & 0.0 & 0 & 0.0 & 3 & 7.5 & 0 & 0.0 \\
\hline Phoenix(F) & 0 & 0.0 & 0 & 0.0 & 0 & 0.0 & 0 & 0.0 \\
\hline Salt Lake City(G) & 3 & 42.8 & 1 & 11.1 & 10 & 25.0 & 3 & 37.5 \\
\hline San Diego(H) & 2 & 28.6 & 3 & 33.3 & 4 & 10.0 & 0 & 0.0 \\
\hline Tucson(I) & 1 & 14.3 & 0 & 0.0 & 1 & 2.5 & 0 & 0.0 \\
\hline All Cities & 7 & 100.0 & 9 & 100.0 & 40 & 100.0 & 8 & 100.0 \\
\hline
\end{tabular}

Table 6 indicates that among job-location subjects, significantly more chose City $\mathrm{H}$ when there was no repository in any city than when City $\mathrm{H}$ contained the repository ( 38 percent vs. 10 percent, $\chi^{2}(1)=7.4, p<.01$ ). However, this result is misleading. The last two rows of Table 6 include those individuals who actually examined technological hazards. Among job-location subjects who examined the technological hazard for City $\mathrm{H}, 43$ percent chose City $\mathrm{H}$ when there was no repository in any city, but only 11 percent chose City $\mathrm{H}$ when it contained the repository. These numbers fail to meet conventional levels of significance, using Fisher's Exact Test $\left(\chi^{2}(1)=2.1\right.$, n.s. $)$. 
TABLE 6

Effects of Repository on Job-Choice Subjects in First Wave of Experiments

Treatments Other cities City $\mathrm{H}$

No. of subjects

29

No. repositories in any city

$18(4)^{\mathrm{a}}$

$11(3)^{\mathrm{a}}$

40

Repositories in City H

$36(8)^{\mathrm{a}}$

$4(1)^{a}$

a. Figure in parentheses is for those who examined technological hazard information.

\section{CONCLUSIONS AND EXTENSIONS}

Based on the first wave of subjects, the results suggest weak evidence that locating a nuclear waste repository in the most popular city drives people to choose another location. Thus, given the limited sample size of the first wave, the presence of a high-level nuclear waste repository appears to have a marginally significant effect.

The increased number of subjects associated with the second and third waves of the experiments allowed two major advances relative to the conclusions drawn on the basis of the first wave. First, based upon the initial evidence obtained from the experiments conducted during 1988 , the results offered weak evidence that locating a nuclear waste repository in a popular city causes people to select some other location. However, due to limited sample size, this result was only marginally significant. The problem was that in order for the repository to make a difference, a subject must have examined the repository card and then selected another city. Increased sample size was necessary in order to get a sufficient number of subjects who actually looked at the repository card.

Second, when surveys are conducted, respondents are frequently given some information regarding the objectives of the survey. Such information could conceivably cause the respondents to be more alert for certain items or issues than otherwise would be the case. For the first two waves of experiments, absolutely no information was provided to the subjects, indicating that the objective of the study was to analyze the consequences of locating a nuclear waste facility close to a major metropolitan area. However, the third wave of subjects was provided with the following instruction: 


\section{INSTRUCTIONS}

This study is about how people decide where they want to live. The purpose of this study is to determine the effect of locating the high-level nuclear waste repository in a western state on people's decisions about where to live. In this study you will make a choice among several possible cities in which you might live.

Click the mouse on the right arrow when you are ready for the next page of instructions.

The question addressed by these instructions is whether subjects, having been alerted to the objectives of the study, would be more likely to examine the technological hazard card containing the repository scenario. The answer is no. Although those who received the special instructions were somewhat more likely to examine the technological hazards information ( 18 of 58 or 31 percent versus 25 of 122 or 20 percent), the difference is not statistically significant $\left(\chi_{1}^{2}=2.4, n . s\right.$.). Evidently, those who are concerned about technological hazards check that information, and those who are not concerned do not check it.

We might then ask among those who did check the technological hazard information for City $\mathrm{H}$, what proportion actually selected City $\mathrm{H}$ as a function of the repository's presence or absence. The following responses were obtained:

a. No repository in City H: 7 of 11, or 64 percent

b. Repository in City $\mathrm{H}$ and normal instructions: 5 of 25 , or 20 percent

c. Repository in City $\mathrm{H}$ and special instructions: 4 of 18 , or 22 percent

Of those who examined the technological hazards information, significantly more selected City $\mathrm{H}$ when it did not have the repository (a vs. b and $\mathrm{c}$ above; $\left.\chi^{2}(2)=7.69, p<.025\right)$. Among those examining the technological hazards information when the repository was in City $\mathrm{H}$, the highlighting instructions had no effect (b vs. $c$ above; $\chi^{2}(1)=0.03$, n.s.). In sum, the presence of the repository makes a difference. Individuals are dissuaded from selecting a location that has a nuclear waste repository in the vicinity, even when the repository scenario reflects a fairly benign series of events as opposed to a major accident.

One of the most noteworthy findings of the study is that employment conditions are a very important consideration in the migration decision process of young people. This finding is consistent with the results of numerous migration studies that use both aggregate migration data and micro data on individuals and households. Although young, job-oriented migrants may be deterred from migrating to Las Vegas by the presence of a nuclear waste repository there, potentially strong effects also could be exerted on them indirectly through employment. If 
accidents or other events should directly reduce employment growth in Las Vegas, an indirect consequence could be to depress in-migration to Las Vegas, which would in turn cause still lower rates of employment growth there, less inmigration, etc.

A preliminary detailed look at the data suggests that the migration decision process can often be characterized as reducing, in the later stages, to a choice between two cities, among which more complete sets of attributes are examined (at least over the western cities that comprised the list of choices).

A possible extension of this study would be to introduce a variety of scenarios for the nuclear waste repository. With these, the sensitivity of the location choice could be tested in relation to stronger, more powerful descriptions with graduation downwards to weaker scenarios and to "signal events" that might be incorporated in these scenarios. The description used in the current data set was not the strongest proposed.

Perhaps more important than the actual findings of this study is the development of a computer protocol that allows migration decisions to be studied in a laboratory experimental setting. Moreover, the methodology developed here can be used to study many types of migration determinants, including natural and man-made hazards, neither of which has previously been examined as a determinant of migration decisions. Finally, with a sufficient number of subjects, the experimental results could be used to estimate a behavioral model. If relevant survey data were also available, perhaps a roughly comparable behavioral model could be estimated using these data. Comparing the empirical results obtained from experimental and survey data could prove very valuable.

Finally, empirical findings for younger, employment-related potential migrants compared to older, retirement-related potential migrants suggest that the two groups behave differently. For example, younger persons are more concerned about technological risks than older persons. This result implies that in analyzing field data separate migration models should be estimated for younger and older persons.

\section{ENDNOTES}

1. For surveys of the determinants of migration, see Greenwood (1975, $1985,1996)$. Some years ago, Wolpert (1966) discussed migration decisions in the context of "environmental stress," which for him includes factors such as "traffic congestion, blight, air and water pollution, lawlessness, lack of open spaces, and noise levels" (p. 97). However, the paper is conceptual and includes nothing of an empirical nature. 
2. The decision components could also be identified empirically by conducting a small-scale survey of individuals making or about to make retirement migration decisions. Because budget limitations prevented us from conducting such a survey, we relied on our knowledge of the migration literature to identify the important decision components.

3. The same small-scale survey mentioned in note 2 could also be used to determine the set of alternative retirement locations typically considered by retirees, but again such a survey was beyond the scope of our study.

4. Such beliefs could also be assssed in a small-scale survey.

5. In our analysis we have studied the order in which subjects consider the available information, but not the amount of time they spend on each piece of information.

6. The various characteristics of each metropolitan area were drawn from several published sources, of which Boyer and Savageau, Places Rated Almanac, Rand McNally and Co., 1985, was the main reference. Other sources were the Statistical Abstract of the United States, Employment and Earnings published by the Bureau of Labor Statistics, and State Government Finances in 1986, published by the U.S. Bureau of the Census.

7. One subject failed to recognize the need to fill in the demographic information and departed before the operator of the experiment realized that some information was missing.

\section{REFERENCES}

Abelson, Robert P., and Ariel Levi. "Decision Making and Decision Theory." In G. Lindzey and E. Aronson (eds.). Handbook of Social Psychology. New York: Random House, 1985.

Anderson, Norman H. Methods of Information Integration Theory. New York: Academic Press, 1982.

Bettman, James R., and C. Whan Park. "Effects of Prior Knowledge and Experience and Phase of Choice Process on Consumer Decision Processes: a Protocol Analysis." Journal of Consumer Research 7 (1980): 234-248.

Einhorn, Hillel J. "The Use of Nonlinear, Noncompensatory Models in Decision Making." Psychological Bulletin 73 (1970): 211-230.

Einhorn, Hillel J., Donald N. Kleinmuntz, and Benjamin Kleinmuntz. "Linear Regression and Process-Tracing Models of Judgment." Psychological Review 86 (1979): 465-485.

Ericsson, K. Anders, and Herbert A. Simon. Protocol Analysis: Verbal Reports as Data. Cambridge, MA: MIT Press, 1984. 
Greenwood, Michael J. "Internal Migration in Developed Countries." In M. R. Rosenzweig and O. Stark (eds.). Handbook of Population and Family Economics. Amsterdam: North-Holland, forthcoming, 1996.

Greenwood, Michael J. "Human Migration: Theory, Models, and Empirical Studies." Journal of Regional Science 25 (1985): 521-544.

Greenwood, Michael J. "Research on Internal Migration in the United States: A Survey." Journal of Economic Literature 13 (1975): 397-433.

Greenwood, Michael J., Peter R. Mueser, David A. Plane, and Alan M. Schlottmann. "New Directions in Migration Research. Perspectives from Some North American Regional Science Disciplines." Annals of Regional Science 25 (1991): 237-270.

Hammond, Kenneth R., Thomas R. Stewart, Berndt Brehmer, and Derick O. Steinmann. "Social Judgment Theory." In M.F. Kaplan and S. Schwartz (eds.). Human Judgment and Decision Processes. New York: Academic Press, 1975.

Montgomery, Herman and Ola Svenson. "On Decision Rules and Information Processing Strategies for Choice among Multiattribute Alternatives." Scandinavian Journal of Psychology 17 (1976): 283-291.

Nickerson, Carol A., and Gary H. McClelland. "Scaling Distortion in Numerical Conjoint Measurement." Journal of Applied Psychological Measurement 8 (1984): 183-198.

Pampel, Fred C., Irwin P. Levin, Jordan J. Louviere, Robert J. Meyer, and Gerard Rushton. "Retirement Migration Decision-Making." Research on Aging 6 (1984): 139-162.

Slovic, Paul and Sarah Lichtenstein. "Comparison of Bayesian and Regression Approaches to the Study of Information Processing in Judgment." Organizational Behavior and Human Performance 6 (1971): 649-744.

Tversky, Amos. "Elimination by Aspects: a Theory of Choice." Psychological Review 79 (1972): 281-299.

Tversky, Amos and Shmuel Sattath. "Preference Trees." Psychological Review 86 (1979): 542-573.

Wolpert, Julian. "Migration as an Adjustment to Environmental Stress." Journal of Social Issues 22 (1966): 92-102. 